\title{
Clinical experiences at veterans administration primary care clinics: An interprofessional education project for advanced practice nurses and health professions students
}

\author{
Margaret Brommelsiek * Jane Anthony Peterson, Sarah Knopf-Amelung, Tracy Lynn Graybill \\ School of Nursing \& Health Studies, University of Missouri-Kansas City, United States
}

Received: April 27, 2017

DOI: $10.5430 /$ jnep.v7n12p1
Accepted: June 28, 2017

Online Published: July 6, 2017

URL: https://doi.org/10.5430/jnep.v7n12p1

\begin{abstract}
There is limited literature that specifically addresses how academic institutions and healthcare facilities effectively establish and manage clinical experiences for students. Since advanced practice nursing education (APRN) programs strive to provide appropriate clinical experiences as part of their students' educational training, it is imperative that academic institutions and clinical facilities establish working relationships and protocols for productive collaboration. Barriers may exist in arranging student clinical placements, including scheduling conflicts and provider workload burden. Collaborative approaches for placing APRN students in primary care settings can be beneficial for student learning and the clinical care of patients. The purpose of this paper is to provide an initial roadmap for coordinating APRN and other health professional students' placement in clinical rotations at a Veterans Health Administration Medical Center (VAMC) primary care clinic in the Midwest.
\end{abstract}

Key Words: Interprofessional education, Nursing, Veterans health

\section{INTRODUCTION}

Meaningful and worthwhile clinical experiences in a variety of settings help prepare advanced practice registered nursing (APRN) students for readiness to practice in primary care settings. ${ }^{[1]}$ For APRN students, the experiential and practical nature of the clinical practicum serves as a counterpart to the theoretical content provided in the classroom and provides an important opportunity for acquiring first-hand knowledge of a patient's lived experience of health. ${ }^{[2]}$ Adequate and appropriate clinical training helps to ensure that future nurse practitioners will deliver high quality, safe patient care,${ }^{[3]}$ as the clinical experience can influence student attitudes both positively and negatively in ways that can carry to professional practice. ${ }^{[4]}$ The clinical environment can be very complex. Yet despite uncertain variables including instability and recurring change, ${ }^{[5]}$ clinical environments also provide students with a number of positive benefits including increased resilience and learning to work as members of teams. ${ }^{[6-8]}$ The majority of literature on clinical experiences for students has been focused on students being adequately prepared in the classroom for their transition to the clinic setting and on the clinical environment's impact on student attitudes. There is, however, limited literature that specifically addresses how academic institutions and healthcare

\footnotetext{
*Correspondence: Margaret Brommelsiek; Email: brommelsiekm@umkc.edu; Address: School of Nursing \& Health Studies, University of Missouri-Kansas City, United States.
} 
facilities effectively establish and manage effective clinical experiences for students. Since APRN programs strive to provide appropriate clinical experiences as part of their students' educational training, it is imperative that both the academic institution and the clinical facility establish a working relationship and protocols for collaboration. The purpose of this paper is to provide an initial roadmap for coordinating APRN and other health professional students' placement in clinical rotations at a Veterans Health Administration Medical Center (VAMC) primary care clinic in the Midwest.

Approximately 30 million individuals gained access to healthcare $^{[9]}$ with the establishment of the Affordable Care Act (ACA) in 2010. As a result, there in an increased demand for health services and healthcare professionals, particularly APRNs. In an effort to meet this demand, educational institutions with APRN programs have increased their enrollments to prepare future advanced practice nurses to function to the full extent of their educational preparation ${ }^{[10]}$ in providing care within a variety of healthcare settings. The increases in patient load in primary care settings have impacted the availability of clinicians to precept students while balancing busy schedules. ${ }^{[11]}$ These factors create challenges for health professional schools to arrange for adequate clinical training sites for their students. ${ }^{[12,13]}$ Subsequently, many APRN graduates may not have the clinical experience and skills necessary to function competently without costly and time-consuming job orientation and training programs with initial employment. ${ }^{[14,15]}$

\subsection{Context}

The education of health professionals must incorporate the clinical care of patients in order to prepare graduates that are ready to practice and lead clinical teams to provide high quality care ${ }^{[16]}$ To accomplish this task, leaders in academic and healthcare institutions must jointly collaborate in the educational endeavors to prepare future health professionals. ${ }^{[3]}$ Advanced practice nursing programs typically strive to create alliances with healthcare partners to provide students with educational clinical learning experiences while students provide hands-on patient care. ${ }^{[17]}$ A number of barriers may exist in arranging student clinical placements, including scheduling conflicts and provider workload burden. In spite of these challenges, collaborative approaches for placing APRN students in primary care settings can be beneficial for student learning and the clinical care of patients.

\subsection{Educational institution barriers and challenges}

Developing relationships between academic institutions and federally operated healthcare facilities, such as the VAMC, may provide greater operational flexibility than privately owned primary care clinics. ${ }^{[18]}$ VAMC primary care clinics serve veterans of various ages with multiple physical and psychosocial problems and may provide excellent learning experiences for APRN and other health professional students. Development of collaborative relationships between academic institutions and VAMC primary care clinics is one strategy to increase the availability of positive clinical learning experiences for health professions students.

From an educational institution perspective, coordination of clinical experiences with healthcare institutions can be challenging. In many APRN programs, there are not enough doctoral faculty to provide the necessary guidance and expertise to support student knowledge acquisition and professional growth. ${ }^{[9]}$ Competition among other health professions schools needing to place their students in clinical rotations is a global phenomenon. According to Papastavrou, Dimitriadou and Tsangar ${ }^{[8]}$ providing quality rotation for nursing students in supportive clinical environments is a primary concern and impacts student satisfaction with their educational training and experience. In the Midwest region where this project occurred, there are currently 26 degree granting nursing programs and three medical schools within the immediate area. Perhaps the greatest challenge facing educational institutions for placing students in clinical rotations is the often lengthy and complex administrative and regulatory requirements of the healthcare institutions. Clinical rotation sites require compliance and documentation of students prior to the rotation that may include background and security checks, drug screenings, and HIPPA and OSHA training. New regulatory requirements regarding patient records may place limitations on student access to data and documentation at some healthcare facilities.

An additional consideration in working with healthcare facilities not associated with the academic institution is the requirement for complex affiliation agreements. In the MultiDiscipline Clerkship/Clinical Training Site Survey, ${ }^{[19]}$ nurse practitioners rated security requirements and legal issues (81\%), student access to health electronic records $(75 \%)$, and other administrative functions such as scheduling, coordination, and completing forms (74\%) as the greatest challenges to developing and sustaining clinical training sites for students. This suggests that it will be increasingly necessary to find ways to eliminate barriers and challenges in coordinating clinical experiences for health professions students.

\subsection{VAMC as clinical partner}

A 2011 White House Report suggested an increased focus on the physical, spiritual and mental health concerns of veterans and their caregivers, as a growing number of veterans are managing Multiple Chronic Conditions (MCC). ${ }^{[20,21]}$ In 
recent years, an increasing number of veterans are choosing to seek care in civilian primary care facilities, rather than VAMC clinics. ${ }^{[22]}$ When collaborating with VAMC primary care clinics, clinical opportunities will offer health professions students unique experiences in a team-based approach to providing care for veterans. Clinical preparation at VAMC clinics will provide students with practical experiences and foster a sensitivity for veterans and their families, regardless of where the veteran chooses to seek care.

In 2010, the Department of Veterans Affairs (VA) redesigned their model of primary care delivery for veterans by placing the patient at the center of care with an interprofessional team to engage and collaborate in healthcare decision-making. ${ }^{\text {[23] }}$ The foundation of the VAMC Patient Aligned Care Teams (PACT) with primary care clinics requires that care is patient driven, team-based, coordinated, comprehensive, and continuous. ${ }^{[23]}$ The PACT interprofessional team generally includes a primary care provider, a registered nurse (RN) care manager, a health technician, and a medical clerk. Other clinicians, such as clinical pharmacists, social workers, and psychologists, supplement the PACT to facilitate care coordination between patients and the primary care team. Evaluation data suggests that the PACT approach to care of veterans has increased communication between providers and patients via electronic messaging and telephone calls and has decreased the number of face-to-face visits patients require. ${ }^{[24]}$ In review of Patient-Centered Models of Care, the VAMC PACT in primary care has reduced Emergency Room visits, hospitalizations, and reduced the costs for care for veterans. ${ }^{[25]}$ When APRN and other health professions students learn to work collaboratively, as graduates they will be prepared to practice in interprofessional teams to improve the quality care patients receive.

The Centers of Excellence in Primary Care Education (CoEPCE) offered health professional trainees opportunities to work in PACTs with health professionals within five VAMC primary care facilities. ${ }^{[23,26]}$ Nurse practitioner (NP) residents at one of the CoEPCE sites rated the value of the teaching and mentoring they received through their VAMC clinical experience higher than NP residents who did not receive this training. VAMC NP residents cited continuity of care, the knowledge and skills gained, and responsibility to patients, as pivotal to their educational training. ${ }^{[23]}$ Following the completion of the 12-month CoEPCE program, the 42 NP residents completed a competency tool and improved significantly ( $p \leq .0001$ ) in all domains, including clinical, interprofessional collaboration, patient-centered care, shared decision-making, sustained relationships, and performance improvement. ${ }^{[27]}$ Although tool psychometrics are not fully established and findings are preliminary, VAMC clinical ex- periences may be a promising approach to prepare APRNs for optimal care delivery within complex health systems and with complex patients.

While each of the five CoEPCE sites utilized a different model of education, all of the sites experienced similar challenges and barriers for gaining access and functioning within the VAMC system. The vast majority of challenges involved operational logistics, communication, and collaboration between the VAMC facilities and the educational institutions. ${ }^{[28]}$ Gilman et al. ${ }^{[3]}$ identified the underlying cause for these difficulties was a result of VA program leaders rarely seeking input from their educational partners prior to implementing new clinical curricula or programming. This suggests that to sustain successful partnerships with VAMC facilities, leadership within the VAMC system and collaborating educational institution must have clear expectations and communication procedures in place prior to placing students in clinical rotations.

There is an increased call for primary care providers to be competent in military culture, with clinical training programs specifically designed to prepare students for working with veterans and their family members. ${ }^{[29]}$ According to Westphal and Convey, ${ }^{[30]}$ nurses who are familiar with military culture are better equipped to deliver culturally appropriate care, especially when their pre-clinical training includes both physical and stress injuries. For individuals without formal military training, negotiating military culture can be difficult, particularly if they are unfamiliar with its language and imposed behaviors. ${ }^{[31]}$ Both the military and nursing professions are guided by an ethos of values including shared beliefs around selflessness and exemplar moral standards. ${ }^{[32]}$ Therefore, including military culture as a core component of APRN primary care instruction will instill confidence in students prior to placement in clinical environments serving this patient population.

\section{METHODS}

In 2014, a School of Nursing and Health Studies (SoNHS) at a Midwest University was funded by an Advanced Practice Nurse Education project grant from the Health Services and Resources Administration (HRSA) to implement an interprofessional education (IPE) project. The clinical practicum designed for this IPE project was within the primary care clinics at the VAMC in the Midwest. Working with graduate APRN, pharmacy, clinical psychology, and social work students, the project sought to improve care delivery to veterans managing multiple chronic physical and behavioral health conditions. ${ }^{[33]}$ The training project included an 8week hybrid course on interprofessional education, military culture, and prevalent chronic conditions among veterans, 
with a simultaneous clinical rotation at a VAMC primary care clinic. The curriculum included didactic and on-line modules, with the course offered beginning in spring 2015, and then each fall and spring semester, concluding in late spring 2017. The specific course content included: (1) military culture taught by military/veteran consultants; (2) veterans and a veteran panel who presented specific military experiences and health issues; (3) multiple chronic conditions and use of the Chronic Care Model; (4) behavioral health disorders common among veterans, including post-traumatic stress disorder (PTSD), substance use disorder (SUD), anxiety and depression and the recommended psychosocial and pharmacological treatments, presented by an expert veterans' clinical psychologist; (5) hypertension and other chronic diseases in relationship to co-morbid behavioral disorders; (6) military sexual trauma and moral distress/ethical issues among military personnel and veterans presented by an experts panel; (7) patient/caregiver roles that impact management of chronic conditions; and (8) psychopharmacology, pain management, and treatment modalities specifically focused on behavioral and physical health disorders common among veterans. ${ }^{[33]}$ This content was supplemented through case studies which allowed students to interact as interprofessional teams and to apply course content to patient cases. The final session provided students the opportunity to practice lessons learned using case scenarios and working in interprofessional teams with standardized patients. Project faculty served as observers, providing 360 feedback to the student teams. ${ }^{[33]}$

\subsection{Process for establishing VAMC clinical placements}

In preparation for placing the students within the VAMC clinics, faculty at the SoNHS met with Nursing Administration at the VAMC to discuss the scope of this IPE project and to assign the interprofessional student teams among available Nursing and other health professional preceptors. Because the VA is a federal facility, the placement of students required compliance with a number of regulations. Health professions students who plan to participate in a clinical practicum at the VAMC must undergo a security clearance process before they can begin attending clinical practicum (see Table 1). This process culminates in acquiring a Personal Identity Verification card (PIV card). Students are required to carry the card when on the VAMC premises. This card provides access to buildings, and most importantly, gives students access to electronic health records.

The security clearance process for the students participating in this project included submission of fingerprints and a background check. As a part of the background check, the students scheduled fingerprinting appointments online, completed a packet of eight forms to be submitted to the respective departmental coordinators (in this instance, the VAMC Department of Nursing Education Coordinator), and assigned online trainings. The core project faculty were responsible for completion and submission of a Training and Qualifications and Verification Letter (TQCVL) for every student. Once the educational institution and student paperwork was completed and submitted to the respective coordinator, the students went through a 4-6 week process of screening. When each student was adjudicated as acceptable, the VAMC designated PIV card sponsor assigned a computer code/number which allowed students to get a PIV card for access to buildings, patients, and computer systems. Lastly, students were required to attend a face-to-face orientation to learn how to use the computer system for documentation. In total the process took eight weeks from student completion of paperwork and fingerprinting appointments to student orientation.

Table 1. VAMC security clearance process for students

- Finger print and background check

- Students complete forms and online training

- Students submit hard copies of paperwork to VAMC Nurse Education Coordinator

- University coordinator/faculty to complete TQCVL Letter for each student

- Student cleared for access, PIV code assigned

- VAMC Nurse Education Coordinator notifies university coordinator of available group orientation times

- Security clearance/student orientation finished

\subsection{Evaluation methods}

To evaluate the effectiveness of the course and clinical rotation, students completed weekly reflection questions and focus groups at the end of each 8-week experience. Reflection questions asked students to describe their involvement in the course and clinical placement each week, including what students learned and taught each other, areas they felt confident in or needed to improve upon, how military culture impacted their patient interactions, how they managed patients with multiple chronic conditions, and examples of interprofessional collaborative practice principles. The focus group questions assessed overall outcomes of the project, including highlights of the course and clinical experience, lessons learned from working with veteran patients, team development, understanding of each other's professional roles, barriers to implementing interprofessional collaborative practice, and how the course and clinical experience could be improved in future semesters. Focus group audio was transcribed. Transcripts and text from weekly reflection questions were analyzed for emerging themes related to students' 
experiences in the course and VAMC clinical placement. The university Institutional Review Board approved this project.

\section{Results}

Across five semesters, 68 students were trained (see Table 2). Despite the administrative barriers and complexities associated with placing students at VA primary care clinics, students participating in the project benefited from the clinical experience in a variety of ways. Focus groups and reflection questions produced four over-arching themes (see Table 3 ): students were exposed to a new patient population, increased their understanding of military culture and veterans' perspectives, gained knowledge about veteran health issues, and were exposed to VA models of care.

Table 2. Student demographics

\begin{tabular}{ll}
\hline & \% (n) \\
\hline Race/Ethnicity (select all that apply) & \\
American Indian/Alaska Native & $2.9 \%(2)$ \\
Asian & $13.3 \%(9)$ \\
Black or African American & $7.4 \%(5)$ \\
Hispanic & $4.4 \%(3)$ \\
Native Hawaiian or other Pacific Islander & $0 \%(0)$ \\
White & $69.1 \%(47)$ \\
More than one race & $7.4 \%(5)$ \\
Gender & \\
Female & $85.3 \%(58)$ \\
Male & $14.7 \%(10)$ \\
Age & \\
$20-29$ & $60.3 \%(41)$ \\
$30-39$ & $20.6 \%(14)$ \\
$40-49$ & $19.1 \%(13)$ \\
\hline
\end{tabular}

Table 3. Impact of students' clinical experience at the VA

\begin{tabular}{l}
\hline Themes \\
\hline Exposure to new patient population \\
Military culture and veterans' perspectives \\
Veteran health issues \\
Exposure to VA models of care \\
\hline
\end{tabular}

\section{Theme 1: Exposure to new patient population}

Students shared that this clinical rotation exposed them to a new patient population. They had little experience working with veterans prior and as a result of participation in this project, they increased their comfort working with veterans.

"I learned more here, because I had minimal experience at the VA, but just knowing that it's a different population then what we experience in regular clinical practice, understanding there is more than just diabetes or heart disease there are other issues that these people have gone through, and need help and cannot get it, if you are the first person they see...there are resources for them."

"I've never worked with any veterans before, well maybe, but it's real quick like a walk in clinic so I might not have known but now I've gone into a couple of patients rooms and I'm in with them by myself and they are very angry automatically. And at first I was like, 'should I go get my preceptor?' but the last two times I just tried to talk to them first and try to reassure them that we can handle it. It has gone much better and then we can talk about what we need to talk about. That is something I would never have done without this experience."

"We all have stories but I love listening to veterans tell theirs. It's an incomparable, humble, moving, emotional, and influential experience."

\section{Theme 2: Military culture and veterans' perspectives} Coursework and clinical experience at the VAMC increased students' comprehension of essential issues impacting veterans such as distinctions in conflict(s) served, improved cognition regarding each veteran's experience as a unique event, and impact of military service on behavioral health. Appreciation of the needs of veterans coupled with a deeper understanding of military culture and its impact assisted students in delivering more appropriate and patient-centered care. Although they increased their knowledge of military culture, students expressed that it would be an ongoing learning process.

"Don't assume that everyone has a positive or negative view of the military or the VA. Everyone has a different experience and you have to find a neutral ground for a treatment plan."

"Understanding when the patients were in service will have an effect on patients' behavior."

"Knowing the culture and the VA perspective on addiction as a disease has been very beneficial."

"Military culture is a learning process as are my other competency areas."

"A patient I saw yesterday served in the Vietnam War and was treated pretty bad when he came home. We gave him a little medal and told him thank you for your service and he was pretty happy about that."

\section{Theme 3: Veteran health issues}

Coursework focused on the unique medical and behavioral health issues facing veterans, which students reinforced 
by their encounters during clinical at the VAMC. Students shared that their knowledge of prevalent veterans' health issues increased, as well as their understanding of the complexities and differences in completing health assessments and treatment plans with veteran patients.

"I am so surprised at how young, 30s and 40s...I mean they have twenty comorbidities. They have it all. If you didn't know their age when you went into the room and you were just looking at their problems you would think you were walking into the room to evaluate a 70 or 80 year old."

"Varied behavioral reactions that the veterans can have from PTSD which can last for years. We saw a range of emotions from a few different patients on the same clinic day - with individualized treatment plans appropriate for each patient."

"Slightly familiar with burn pits but not extremely competent on what that situation was like for the veterans and what health consequences to assess for."

"A VA patient with multiple mental health disorders struggles to take care of his other chronic diseases."

\section{Theme 4: Exposure to VAMC models of care}

Clinical experience at the VAMC exposed students to new models of team-based primary care and the VAMC healthcare system in general. Students had the opportunity to work in the PACT model, which echoed the interprofessional collaborative practice model that students were learning in coursework. Students also gained experience helping patients navigate the complex VAMC healthcare system and using specific communication techniques employed by VAMC preceptors.

"Substance abuse and dermatological issues are briefly assessed and referred to mental health and dermatology respectively at the VA whereas in civilian practice these concerns (assuming nothing is emergent) would be further assessed and treatment would be initiated by the PCP. Only upon failure of PCP process would referral to specialists be implemented."

"How the VA utilizes the PACT approach to help drive the patient care so all the issues are addressed for the patient, in a patient-centered model."
"Preceptors provided information on health literacy and communication techniques with specific patient population."

\section{Discussion}

The demand for quality clinical rotation experiences for nursing and other health professions students continues to be challenging, particular in the VA system. Yet, few studies have addressed the process for establishing these rotations. In similar studies with clinical placements of APRN and other health professions students working with veterans, the models used were transformative structures and curricula that emphasized patient-centeredness through shared decisionmaking and collaborative practice and the need for flexibility in delivering a shared-learning experience. ${ }^{[27]}$ In a follow-up to the Rugen 2013 study of the CoEPCE's, ${ }^{[34]}$ an assessment tool was developed to measure student progress. Whereas the overall findings of the study were positive, students reported a need to expand the curricula around several aspects of military culture including, posttraumatic stress disorder, military sexual trauma, and traumatic brain injury prior to their rotations in the VA facility. ${ }^{[34]}$ In a study addressing the needs of social work students working with veterans, ${ }^{[29]}$ several areas of increased need prior to clinical placement include forming relationships, especially the building of trust and rapport with patients; greater understanding of military culture; and a need for specific health issue of veterans such as psychological and behavioral disorders. In comparison, our project included comprehensive curriculum relating to military culture and veteran health needs to equip students with sufficient baseline knowledge to participate in a practicum at the VAMC. As a result, students in our project benefited through several learning dimensions, including exposure to a new patient population, increased understanding of military culture and veterans' perspectives, improved knowledge of veteran health issues, and exposure to new VA models of healthcare.

However, we encountered a number of barriers through our clinical placement of APRN students that required a great deal of flexibility and persistence from the faculty and students involved. The security clearance process at the VAMC is rigorous and takes a lengthy time, often six to eight weeks. Changes in VAMC procedures or other issues influencing student clinical placements were not promptly communicated which made it difficult to adapt or solve the problem. In addition, scheduling fingerprint and PIV card appointments often did not align with pre-arranged scheduled times with preceptors. Lastly, scheduling a single orientation time in which all of the students were required to attend as a group required very busy students to be flexible and patient. Consideration 
of how the security clearance process would need to work from an educational institution and student perspective were key in the development of successful clinical practicums for the nursing students in this project.

Despite these challenges, we learned that establishing solid relationships with healthcare institutions well in advance of student placement facilitated and strengthened negotiating and resolving these issues as quickly as possible. This project accomplished its desired goal of providing APRN and other health professions students with an opportunity to work within a structured VAMC primary care PACT model. The initial open and collaborative relationship developed between VAMC Nursing Service and project faculty provided open communication that closely aligned with interprofessional clinical practice and the PACT approach to care. Meetings between the key clinical staff at the VAMC and University project faculty occurred twice each semester. This significantly facilitated communication as well as understanding the optimal approach for securing students' clearance and the required documentation and placements within the clinical setting. Perhaps the most valuable lessons learned were to allow ample time to work within a complicated federal healthcare system and assigning a liaison to be the primary contact between the university and the VAMC. Flexibility and a positive attitude were helpful as staff positions changed and the most well intended plans often needed to be re-negotiated.

\section{RECOMMENDATIONS}

Access to care for many US individuals has increased because of the Affordable Care Act. However, the increasing number of patients seeking healthcare has outpaced the number of health professionals available to meet this demand. Challenges exist for health education institutions and healthcare facilities to meet the complex health needs of this growing population. The need to adequately educate and prepare health professionals for readiness to practice requires unique and effective collaboration between academia and healthcare facilities. Health professions schools have increased the number of students enrolled, creating limited hands-on clinical experiences and a competitive shortage for placement of students in healthcare facilities. Furthermore, it has become extremely important to increase quality and efficiency of care to meet the population needs. Interprofessional education for health professions students and actively engaging students in learning best practices is critical for the transformation of healthcare delivery and quality. ${ }^{[35-37]}$

Interprofessional education with clinical practicum is an important part of preparing APRNs for readiness to practice at the full extent of their educational preparation and for collaboration in healthcare teams. The VAMC's PACT model of primary care provides a beneficial learning environment for health professions students because it not only serves a broad and diverse patient population, but functions based on interprofessional collaboration. The PACT has a structure in which students have opportunities to engage in patient centered team-based care delivery. VAMC clinical rotations focusing on complex behavioral and physical conditions can provide rich learning experiences for APRN students that are applicable to the growing veteran population, as well as the general US population.

\section{Limitations}

This study was focused on APRN students and health professions students from pharmacy, social work and clinical psychology working with veterans at a veterans' administration primary care clinic. The majority of students who participated over the three years of the project were primarily female and white, which is representative of three of the four health professions areas at our university. Each VAMC is unique, therefore all of the strategies employed during this project may not be applicable to other VAMC as each has its own processes for collaborating with outside institutions. Lastly, the preceptors assigned to this project from the VAMC were deeply engaged throughout the project, even attending some of the faculty and student training sessions. While this commitment from the preceptors was a critical element in regard to the success of this particular project, this may not be the case working with preceptors at other VAMC facilities.

\section{Conclusion}

APRN and other health professional students engaged in this VAMC 8-week training project met many challenges, yet many successes. The project director and faculty learned to navigate the VAMC system requirements and negotiate strategies to achieve and maintain optimal clinical learning experiences for the students. Although the VAMC competency too ${ }^{[33]}$ was not available at the onset of this experience, student focus group data and reflection questions and informal faculty and VAMC clinical APRN preceptors' feedback have provided formative and summative evaluation. Focus group themes support the continuation and further development of this VAMC training. Health professions students shared their appreciation of learning about veterans' health issues, military culture, veterans' perspectives, and exposure to the VAMC model of care. Although, there was limited outcome data derived from this initial project, focus group data does support further development and engagement in clinical experiences for APRN students within VAMC primary care clinics. 


\section{ACKNOWLEDGEMENTS}

This work was supported by D09HP26956 an Advanced Nursing Education Grant from the Health Services and Re- sources Administration.

\section{Conflicts of InTERest Disclosure}

The authors declare that there is no conflict of interest.

\section{REFERENCES}

[1] Fitzgerald C, Kantrowitz-Gordon I, Katz J, et al. Advanced practice nursing education: challenges and strategies. Nursing Research and Practice. 2012.

[2] Jamshidi N, Molazem Z, Sharif F, et al. The Challenges of Nursing Students in the Clinical Learning Environment: A Qualitative Study. The Scientific World Journal. 2016. PMid:27366787 https://doi.org/10.1155/2016/1846178

[3] Gilman SC, Chokshi DA, Bowen JL, et al. Connecting the dots: Interprofessional health education and delivery system redesign at the Veterans Health Administration. 2014

[4] Tiwaken SU, Caranto LC, David JJT. The real world: lived experiences of student nurses during clinical practice. International Journal of Nursing Science. 2015; 5(2): 66-75.

[5] Baraz S, Memarian R, Vanaki Z. Learning challenges of nursing students in clinical environments: A qualitative study in Iran. Journal of Education and Health Promotion. 2015

[6] Howe A, Smajdor A, Stöckl A. Towards an understanding of resilience and its relevance to medical training. Medical Education. 2012; 46(4): 349-356. PMid:22429170 https ://doi.org/10.1 111/j.1365-2923.2011.04188.x

[7] Hood K, Cant R, Leech M, et al. Trying on the professional self: nursing students' perceptions of learning about roles, identity and teamwork in an interprofessional clinical placement. Applied Nursing Research. 2014; 27(2): 109-114. PMid:24050916 https://doi.org/10.1016/j.apnr.2013.07.003

[8] Papastavrou E, Dimitriadou M, Tsangari H, et al. Nursing students' satisfaction of the clinical learning environment: a research study. BMC Nursing. 2015; 15(1): 44. PMid:27436992 https: //doi.org/10.1186/s12912-016-0164-4

[9] American Association of Colleges of Nursing. White paper on reenvisioning the clinical education of advanced practice registered nurses. 2015.

[10] Iglehart JK. Expanding the role of advanced nurse practitioners-risks and rewards. New England Journal of Med. 2013; 368: 1935-1941. https://doi.org/10.1056/NEJMhpr1301084

[11] Burns C, Beauchesne M, Ryan-Krause P, et al. Mastering the preceptor role: challenges of clinical teaching. Journal of Pediatric Health Care. 2006; 20(3): 172-183.

[12] Leners D, Sitzman K, Hessler KL. Perceptions of nursing student clinical placement experiences. International Journal of Nursing Education Scholarship. 2016.

[13] Yonge O, Myrick F, Haase M. Student nurse stress in the preceptorship experience. Nurse Educator. 2002; 27(2): 84-88.

[14] Reiter MA, Young A, Adamson C. Decrease new graduate orientation costs by using HESI exit exam scores. Journal of Nursing Administration. 2007; 37(10): 459-463.

[15] Rich KL, Nugent KE. A United States perspective on the challenges in nursing education. Nurse Education Today. 2010; 30(3): 228-232.

[16] Knebel E, Greiner AC. (Eds.). Health professions education: A bridge to quality. National Academies Press; 2003.
[17] Ellison G. Blowing open the bottleneck in Oklahoma. Oklahoma Nurse. 2008; 53(3): 16

[18] Dobalian A, Bowman CC, Wyte-Lake T, et al. The critical elements of effective academic-practice partnerships: a framework derived from the Department of Veterans Affairs Nursing Academy. BMC Nursing. 2014; 13(1): 36.

[19] Erikson C, Hamann R, Levitan T, et al. Recruiting and maintaining US clinical training sites: joint report of the 2013 multi-discipline clerkship/clinical training site survey. 2014.

[20] White House. Strengthening our Military Families. Interagency Policy Committee (IPC). 2011.

[21] Weiss KB. Managing complexity in chronic care: an overview of the VA state-of-the-art (SOTA) conference. Journal of General Internal Medicine. 2007; 22(3): 374-378.

[22] Luby CD. Promoting military cultural awareness in an off-post community of behavioral health and social support service providers. Advances in Social Work. 2012; 13(1): 67-82.

[23] Rugen KW, Watts SA, Janson SL, et al. Veteran affairs centers of excellence in primary care education: transforming nurse practitioner education. Nursing Outlook. 2014; 62(2): 78-88.

[24] Rosland AM, Nelson K, Sun H, et al. The patient-centered medical home in the Veterans Health Administration. The American Journal of Managed Care. 2013; 19(7): e263-72.

[25] Grumbach K, Grundy P. Outcomes of implementing patient centered medical home interventions. Washington, DC: Patient-Centered Primary Care Collaborative. 2010.

[26] Kashner TM, Hettler DL, Zeiss RA, et al. Has interprofessional education changed learning preferences? A national perspective. Health Services Research. 2016.

[27] Rugen KW, Speroff E, Zapatka SA, et al. Veterans Affairs Interprofessional Nurse Practitioner Residency in Primary Care: A Competencybased Program. The Journal for Nurse Practitioners. 2016; 12(6): e267-e273

[28] Swenty CL, Schaar GL, Butler RM. An academic-VA partnership: Student interprofessional teams integrated with VA PACT teams. 2016.

[29] Linn B, Butler L, Bruce S, et al. On working with veterans: what social work and nursing students need to know. Journal of Military and Veterans Health. 2015; 23(3): 5.

[30] Westphal RJ, Convoy SP. Military culture implications for mental health and nursing care. Online Journal of Issues in Nursing. 2015; 20(1): 4.

[31] Meux A. Culture clashes: Frustrations of military behavioral health providers. Dallas News. 2016. Available from: https ://www . dallasnews. com/opinion/commentary/2016 /11/18/culture-clashes-frustrations-military-behav ioral-health-providers

[32] Convoy S, Westphal RJ. The importance of developing military cultural competence. Journal of Emergency Nursing. 2013; 39(6): 591.

[33] Peterson JA, Brommelsiek M, Knopf-Amelung S. An interprofessional education project to address veterans' healthcare needs. International Journal of Higher Education. 2017; 6(1): 1-16. 
[34] Rugen KW, Speroff E, Zapatka SA, et al. Veterans Affairs Interprofessional Nurse Practitioner Residency in Primary Care: A Competencybased Program. The Journal for Nurse Practitioners. 2016; 12(6): e267-e273.

[35] Interprofessional Education Collaborative Expert Panel. Core competencies for interprofessional collaborative practice: Report of an expert panel. Washington, DC: Interprofessional Education Collabo- rative. 2011

[36] Institute of Medicine. Learning to improve health from interprofessional models across the continuum of education to practice: Workshop summary. Washington, D.C.: National Academy Press; 2013.

[37] World Health Organization. Framework for action on interprofessional education \& collaborative practice. Geneva: World Health Organization. 2010. 